\title{
Chandra detection of diffuse X-ray emission from the globular cluster Terzan 5
}

\author{
P. Eger ${ }^{1}$, W. Domainko ${ }^{2}$, and A.-C. Clapson ${ }^{2}$ \\ 1 Erlangen Centre for Astroparticle Physics, Universität Erlangen-Nürnberg, Erwin-Rommel-Str. 1, 91058 Erlangen, Germany \\ e-mail: peter.eger@physik.uni-erlangen.de \\ 2 Max-Planck-Institut für Kernphysik, PO Box 103980, 69029 Heidelberg, Germany
}

Received 24 November 2009 / Accepted 19 January 2010

\section{ABSTRACT}

\begin{abstract}
Aims. Terzan 5, a globular cluster (GC) prominent in mass and population of compact objects, is searched for diffuse X-ray emission, as proposed by several models.

Methods. We analyzed the data of an archival Chandra observation of Terzan 5 to search for extended diffuse X-ray emission outside the half-mass radius of the GC. We removed detected point sources from the data to extract spectra from diffuse regions around Terzan 5. The Galactic background emission was modeled by a 2-temperature thermal component, which is typical for Galactic diffuse emission.

Results. We detected significant diffuse excess emission above the particle background level from the whole field-of-view. The surface brightness appears to be peaked at the GC center and decreases smoothly outwards. After the subtraction of particle and Galactic background, the excess spectrum of the diffuse emission between the half-mass radius and $3^{\prime}$ can be described by a powerlaw model with photon index $\Gamma=0.9 \pm 0.5$ and a surface flux of $F_{\mathrm{x}}=(1.17 \pm 0.16) \times 10^{-7} \mathrm{erg} \mathrm{cm}^{-2} \mathrm{~s}^{-1} \mathrm{sr}^{-1}$ in the $1-7 \mathrm{keV}^{\mathrm{band}}$. We estimated the contribution from unresolved point sources to the observed excess to be negligible. The observations suggest that a purely thermal origin of the emission is less likely than a non-thermal scenario. However, from simple modeling we cannot identify a clearly preferred scenario.
\end{abstract}

Key words. globular clusters: individual: Terzan 5 - acceleration of particles - X-rays: diffuse background

\section{Introduction}

Terzan 5 (stellar luminosity $L_{\text {star }}=1.5 \times 10^{5} L_{\odot}$, Harris 1996) is the Galactic globular cluster (GC) with the largest population of known millisecond pulsars (33 MSPs, Ransom 2008). Furthermore, a high production rate for low-mass X-ray binaries (LMXBs) is expected in the extremely dense core of Terzan 5 (Ivanova et al. 2005). The GC is located at a distance of $5.5 \mathrm{kpc}$ (Ortolani et al. 2007) or $8.7 \mathrm{kpc}$ (Cohn et al. 2002), only $\sim 1.7 \mathrm{deg}$ above the Galactic plane (RA: $17^{\mathrm{h}} 48^{\mathrm{m}} 04^{\mathrm{s}} 0$, Dec: $\left.-24^{\circ} 46^{\prime} 45^{\prime \prime}\right)$. Its core $\left(r_{\mathrm{c}}\right)$ and half-mass radii $\left(r_{\mathrm{h}}\right)$ are $0.18^{\prime}$ and $0.83^{\prime}$, respectively (Harris 1996).

GCs are expected to contain intracluster gas originating from the mass loss of evolved stars. Since GCs move through the Galactic halo medium with typical velocities of $\sim 200 \mathrm{~km} \mathrm{~s}^{-1}$, bow shocks should form in front of them in the direction of their proper motion (Krockenberger \& Grindlay 1995). These shocks have the ability to both accelerate particles and heat the gas behind them. In this scenario, electrons accelerated at the bow shock could produce diffuse non-thermal X-ray emission because of either inverse Compton (IC) scattering on ambient photon fields (Krockenberger \& Grindlay 1995) or non-thermal bremsstrahlung resulting from the deflection of the electrons by inter-stellar medium (ISM) nuclei (Okada et al. 2007, henceforth Ok07). Also, diffuse thermal X-ray emission can be emitted from shock-heated material trailing behind the moving GC. For a detailed discussion of the bow-shock scenario, see Ok07.

The first unresolved diffuse X-ray emission from GCs (47 Tuc, $\omega$ Cen, and M 22) was reported by Hartwick et al. (1982) using the Einstein observatory, which was later confirmed by Krockenberger \& Grindlay (1995) with ROSAT. Recently, using Chandra data, Ok07 detected significant diffuse X-ray emission from the GCs 47 Tuc, NGC 6752, M 5, and $\omega$ Cen. They find that the diffuse source at the position of $\omega$ Cen is likely to be a background cluster of galaxies. A similar extraGalactic nature is proposed for 47 Tuc by Yuasa et al. (2009). The remaining potentially GC-associated diffuse X-ray emission, from M 5 and NGC 6752, could arise from different scenarios. The emission in M 5 features an arclike morphology and exhibits a thermal spectrum $(k T<0.1 \mathrm{keV})$, possibly from shock-heated gas. The clumpy structure seen near NGC 6752 presents a hard non-thermal spectrum $(\Gamma \sim 2)$ and a radio counterpart, maybe from non-thermal bremsstrahlung emission by shock-accelerated electrons hitting nearby gas clouds.

Non-thermal emission in the X-ray band may also be associated with compact objects, either directly, as detected from isolated low-mass X-ray binary systems (see for instance Wijnands et al. 2005, for such a candidate system in Terzan 5), or as secondary emission from the population of high-energy particles they would generate. This was modeled for millisecond pulsars by Venter \& de Jager (2008, henceforth VJ08) for the synchrotron radiation mechanism. The second case may translate into larger physical scales, due to the diffusion of the energetic particles away from their source. The populations of compact objects in Terzan 5 may provide an opportunity to test these scenarios. Apparent, extended X-ray emission from the direction of GCs might also arise from a population of faint unresolved point-like sources below the detection limit of the observing instrument.

In this work we analyzed an archival Chandra observation to search for a diffuse emission component above the Galactic background associated with Terzan 5 . We characterized the 
X-ray signal using spatially resolved spectral analyses, after a careful study of the diffuse Galactic background, and briefly discuss different scenarios for the emission.

\section{X-ray analysis and results}

\subsection{Observation and data preparation}

To search for extended diffuse X-ray emission from Terzan 5, we analyzed the Advanced CCD Imaging Spectrometer (ACIS, Garmire et al. 2003) data of an archival $40 \mathrm{ks}$ Chandra (Weisskopf et al. 2002) observation (ObsID 3798), which was originally performed to characterize the faint X-ray point-source population of this GC (Heinke et al. 2006, henceforth H06). Only the ACIS-S3 chip was switched on, so we were only able to search for diffuse X-ray emission at angular distances $\$ 4^{\prime}$ from the cluster core. A comprehensive study of diffuse X-ray emission seen from a number of Galactic GCs was performed by Ok07. However, these authors excluded Terzan 5 from their work because the only available Chandra dataset at that time suffered from serious pile-up effects because of a bright binary outburst in the field-of-view (FoV). In this paper we analyzed data from a newer observation where such an event did not occur.

For the X-ray analysis we used the CIAO software version 4.1, supported by tools from the FTOOLS package and XSPEC version 12.5.0 for spectral modeling (Arnaud 1996). The eventl data were reprocessed with the latest position and energy calibration (CTI correction, v4.1.3) using bad pixel files generated by acis_run_hotpix. The good-time-interval (GTI) file supplied by the standard processing, which was used by H06, screens out a $\sim 4.0 \mathrm{ks}$ interval of strong background flaring at the end of the observation. To remove an additional time period of $4.3 \mathrm{ks}$ with a slightly increased background level, we used the light curve in the $0.5-7.0 \mathrm{keV}$ energy band after the core region of the cluster and additional bright sources were removed from the data. A screening threshold of $1.0 \mathrm{cts} / \mathrm{s}$ yielded a net exposure of $31.0 \mathrm{ks}$. We chose these stricter criteria with respect to H06 because understanding the background is crucial for analyzing faint extended sources.

\subsection{Extraction regions}

To detect and remove point-like X-ray sources from the eventlist, we ran wavdetect on the GTI-screened dataset in three energy bands (0.5-2.0 keV, 2.0-7.0 keV and 0.5-7.0 keV). H06 used $p$ wdetect for the detection within $r_{\mathrm{h}}$ and wavdetect for outer regions. In this paper we only analyzed areas outside $r_{\mathrm{h}}$, so results should be comparable. We estimated a point-source detection limit of $\sim 2 \times 10^{-15} \mathrm{erg} \mathrm{cm}^{-2} \mathrm{~s}^{-1}$ in the $0.5-7.0 \mathrm{keV}$ band. For the most part our results are compatible with the sources listed by H06, Table 2. However, the shorter exposure time compared to the analysis of H06 led to a higher point-source detection threshold. Therefore we did not detect the faintest seven sources from H06 that we introduced manually into our source list. Sources were removed from the dataset using the $3 \sigma$ radius of the point spread function. Additionally, all events within $r_{\mathrm{h}}$ were disregarded.

To measure the level of diffuse X-ray emission around Terzan 5, we extracted spectra from eight concentric annular regions centered on the cluster core with radii from $1.1^{\prime}$ to $3.9^{\prime}$ (Fig. 1). Each ring has a width of $0.4^{\prime}$. We chose rings with equal width over rings with constant area to have comparable statistical quality in the spectra since the surface brightness decreases with distance from the GC. For the spectral analysis, we

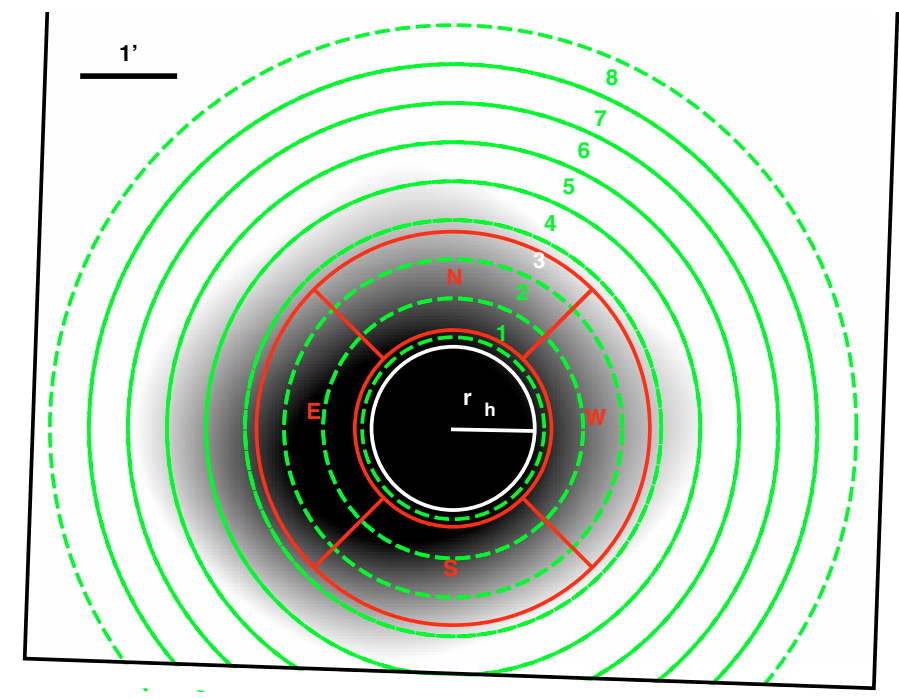

Fig. 1. Smoothed, exposure corrected and NXB subtracted Chandra image of diffuse X-ray emission in the $1-7 \mathrm{keV}$ band around Terzan 5 . Excluded regions from point sources and the region inside $r_{\mathrm{h}}$ (white circle labeled $r_{\mathrm{h}}$ ) were refilled (see text). The wings seen towards the north, east and west are only marginally significant and might be artifacts of the smoothing algorithm. The FoV for this observation is drawn as a box (black). Shown are the eight annular extraction regions (dashed green lines, numbered 1 to 8 ) and the four pie-shaped regions (solid red lines, labeled N, E, S and W). The color scaling is linear and chosen such that the Galactic diffuse level is saturated as white. Thus only emission above the Galactic diffuse level appears in gray scales.

chose the 1-7 keV energy band. Widening the band in either direction lead to lower signal-to-noise ratios. At lower energies an increased contribution to the signal from soft thermal Galactic diffuse emission is expected. At energies above $7-8 \mathrm{keV}$ the charged particle induced background component increases significantly for instruments onboard Chandra. The mean effective area and energy response for each spectrum was calculated by weighting the contribution from each pixel by its flux using a detector map in the same energy band. To subtract the particle induced non-X-ray background (NXB), we used a background dataset provided by the calibration database, where the detector was operated in stowed position. The background spectrum for each ring was extracted from the respective region in this background dataset. To account for the time dependence of the $\mathrm{NXB}$, we scaled the background by the ratio of the source and background count rates in the 9-12 keV energy band for each spectrum (as described by Markevitch et al. 2003).

To produce an image of diffuse X-ray emission above the particle background from the direction of Terzan 5, we extracted counts in the 1-7 keV energy band and refilled the excluded source regions and the region inside $r_{\mathrm{h}}$ with $d m f$ filth using the photon distributions from rings around the excluded areas. We subtracted the particle background using the respective image from the stowed dataset after correction for the different exposures. The resulting image was corrected for relative exposure and adaptively smoothed with asmooth. We required a minimum significance of $3 \sigma$ for the kernel size of the smoothing algorithm. The resulting smoothing radii were a few arcminutes, so that no details smaller than that scale can be seen in the smoothed image. Figure 1 shows the resulting image together with all extraction regions that we used in this work.

Even though a significant contribution from thermal Galactic diffuse emission is expected, the spectra from the single rings 
Table 1. Extraction regions and results from spectral fitting.

\begin{tabular}{|c|c|c|c|c|c|c|c|c|}
\hline Region & $\begin{array}{l}\text { Distance range }{ }^{(1)} \\
(\operatorname{arcsec})\end{array}$ & $\begin{array}{l}\text { Angular range }{ }^{(2)} \\
(\mathrm{deg})\end{array}$ & Excess counts ${ }^{(3)}$ & $\begin{array}{l}\text { NEI: } F_{\mathrm{x}, \text { surf }}{ }^{(4)} \\
\left(10^{-7} \mathrm{erg} \mathrm{cm}^{-2} \mathrm{~s}^{-1} \mathrm{sr}^{-1}\right)\end{array}$ & $\begin{array}{l}\text { NEI: } k T^{(5)} \\
(\mathrm{keV})\end{array}$ & $\begin{array}{l}\text { PL: } F_{\mathrm{x}, \text { surf }}{ }^{(6)} \\
\left(10^{-7} \mathrm{erg} \mathrm{cm}^{-2} \mathrm{~s}^{-1} \mathrm{sr}^{-1}\right)\end{array}$ & PL: $\Gamma^{(7)}$ & $\chi_{\nu}^{2}$ (d.o.f.) \\
\hline Ring 1 & $55-79$ & $0-360$ & $195.2 \pm 26.5$ & - & - & $1.01 \pm 0.25$ & $1.8 \pm 0.4$ & $-/ 1.3(15)$ \\
\hline Ring 2 & 79-103 & $0-360$ & $278.6 \pm 31.1$ & - & - & $0.91 \pm 0.17$ & $2.1 \pm 0.5$ & $-/ 0.7(26)$ \\
\hline Ring 3 & $103-126$ & $0-360$ & $274.8 \pm 33.3$ & - & - & $0.64 \pm 0.14$ & $2.2 \pm 0.6$ & $-/ 1.2(27)$ \\
\hline Ring 4 & $126-150$ & $0-360$ & $259.9 \pm 34.2$ & - & - & $0.47 \pm 0.09$ & $3.0 \pm 0.8$ & $-/ 0.9(24)$ \\
\hline Ring 5 & $150-174$ & $0-360$ & $276.7 \pm 35.3$ & - & - & $0.44 \pm 0.08$ & $2.9 \pm 0.7$ & $-/ 1.4(26)$ \\
\hline Ring 6 & $174-198$ & $0-360$ & $192.7 \pm 34.7$ & - & - & $0.30 \pm 0.10$ & $2.8 \pm 0.8$ & $-/ 0.9(19)$ \\
\hline Ring 7 & $198-222$ & $0-360$ & $227.5 \pm 36.2$ & - & - & $0.26 \pm 0.08$ & $3.6 \pm 1.2$ & $-/ 1.2(22)$ \\
\hline Ring 8 & $222-246$ & $0-360$ & $240.3 \pm 37.5$ & - & - & $0.31 \pm 0.07$ & $3.5 \pm 1.2$ & $-/ 1.0(23)$ \\
\hline \multirow[t]{2}{*}{ Inner } & $55-174$ & $0-360$ & $1273.5 \pm 115.8$ & $1.34 \pm 0.14(\mathrm{soft})$ & 0.59 (soft) & $1.17 \pm 0.16$ & $0.9 \pm 0.5$ & $1.1(52) /$ \\
\hline & & $0-360$ & & $5.36 \pm 0.80$ (hard) & 5.0 (hard) & - & - & $1.3(50)$ \\
\hline \multirow[t]{2}{*}{ Outer } & $175-246$ & $0-360$ & $825.3 \pm 62.5$ & $0.58 \pm 0.19(\mathrm{soft})$ & 0.59 (soft) & - & - & $1.2(36) /-$ \\
\hline & & $0-360$ & & $2.1 \pm 0.7$ (hard) & 5.0 (hard) & - & - & \\
\hline North & $60-120$ & $45-135$ & $191.0 \pm 26.4$ & - & - & $1.44 \pm 0.63$ & $1.5 \pm 1.0$ & $-/ 1.0(45)$ \\
\hline East & $60-120$ & $135-225$ & $176.1 \pm 26.7$ & - & - & $2.10 \pm 0.70$ & $0.6 \pm 0.7$ & $-/ 0.9(44)$ \\
\hline South & $60-120$ & $225-315$ & $230.1 \pm 27.0$ & - & - & $2.46 \pm 0.72$ & $0.8 \pm 0.7$ & $-/ 0.8(47)$ \\
\hline West & $60-120$ & $315-45$ & $165.2 \pm 27.1$ & - & - & $2.10 \pm 0.75$ & $1.7 \pm 1.1$ & $-/ 1.0(45)$ \\
\hline
\end{tabular}

Notes. ${ }^{1}$ Inner and outer radii of the region. ${ }^{2}$ Angular range of the region. ${ }^{3}$ Excess counts after background subtraction. ${ }^{4}$ Intrinsic $0.7-10.0 \mathrm{keV}$ surface flux of the two thermal components. ${ }^{5}$ Temperatures of the two thermal components. ${ }^{6}$ Surface flux $(1-7 \mathrm{keV})$ resulting from an absorbed power-law fit. ${ }^{7}$ Spectral index resulting from an absorbed power-law fit.

were fit well enough by an absorbed power-law model for a preliminary flux estimate. The resulting fit parameters are given in Table 1 . We found significant diffuse excess emission above the particle background in all rings and derived the surface brightness for each region by dividing the model flux by the effective extraction area, which is the geometric ring area inside the FoV minus excluded regions and bad pixels. Due to limited statistics, we fixed the column density at a default value of $N_{\mathrm{H}}=1 \times 10^{22} \mathrm{~cm}^{-2}$. Therefore, we list the observed surface fluxes in Table 1, as opposed to the intrinsic fluxes we provide for all other spectra. The diffuse surface flux shows a clear radial dependence (Fig. 2), which indicates that a significant part of the excess is connected to the cluster. At distances greater than $\sim 170^{\prime \prime}$ from the GC core, the observed surface flux seems to reach a base level of $F_{\mathrm{x} \text {,surf }} \approx 1.5 \times 10^{-18} \mathrm{erg} \mathrm{cm}^{-2} \mathrm{~s}^{-1} \mathrm{arcsec}^{-2}$ (1-7 keV). In the following section we derive the unabsorbed surface flux for the outer region by applying a more realistic physical model.

\subsection{Galactic diffuse background}

Terzan 5 is close to the Galactic plane where diffuse Galactic emission becomes an important component. However, the Chandra blank-sky datasets are composed of observations towards high Galactic latitudes, which would underestimate the sky background in our case. To test whether the spectrum observed from the outer three rings is compatible with thermal Galactic diffuse emission, we used a more physically reasonable model. Similar to Kaneda et al. (1997) and Ebisawa et al. (2005), who modeled the diffuse Galactic ridge emission as observed with ASCA and Chandra, respectively, we describe the Galactic diffuse component using a two-temperature (2-T) nonequilibrium ionization model (NEI) (Masai 1984). To improve the statistical quality, we combined the outer three (175-246") rings into a single spectrum. The spectrum was adaptively binned to a minimum of 20 excess counts per bin. As background we again used the spectrum extracted from the same region in the NXB dataset. We fitted a 2-T NEI model to the outer spectrum, freezing most of the parameters to the best-fit

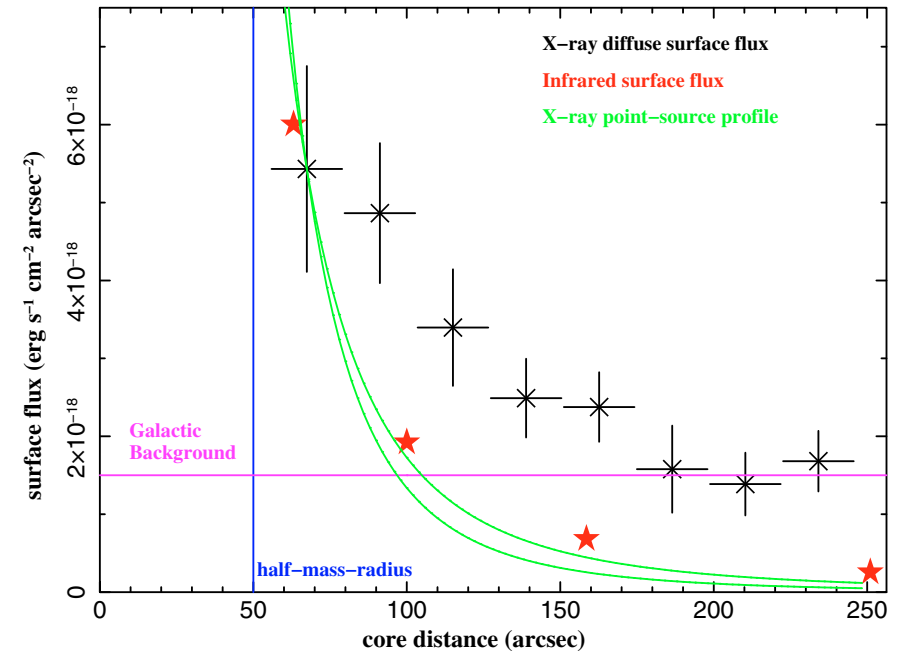

Fig. 2. Radial dependence of the observed diffuse X-ray surface flux above the particle background in the $1-7 \mathrm{keV}$ band as seen with Chandra (black crosses with error bars). Stars (red) denote the infrared surface brightness profile from Trager et al. (1995). The solid curves (green) show the X-ray point-source distribution described by a generalized King-profile (Heinke et al. 2006) for the two extreme cases $q=1.43 \pm 0.11$. All profiles are scaled to match the first diffuse X-ray data point, using an exponential fit in the case of the infrared data. The vertical (blue) and the horizontal (magenta) line denote $r_{\mathrm{h}}$ (Harris 1996) and the Galactic diffuse background level, respectively.

values from Table 8 in Ebisawa et al. (2005). We left the surface brightnesses of the two components and the $\mathrm{N}_{\mathrm{H}}$ free to vary to account for the difference in flux and column density between the region around Terzan 5 and the area observed by Ebisawa et al. (2005). In addition, we allowed the Si-abundance of the soft component as a free fit-parameter, because the low-ionized Si line at $\sim 1.8 \mathrm{keV}$ (Kaneda et al. 1997; Ebisawa et al. 2005) was otherwise underestimated.

The spectrum of the outer region together with the model fit is shown in Fig. 3 (Top). To be able to compare our results to the analysis of Ebisawa et al. (2005), we chose an energy range 

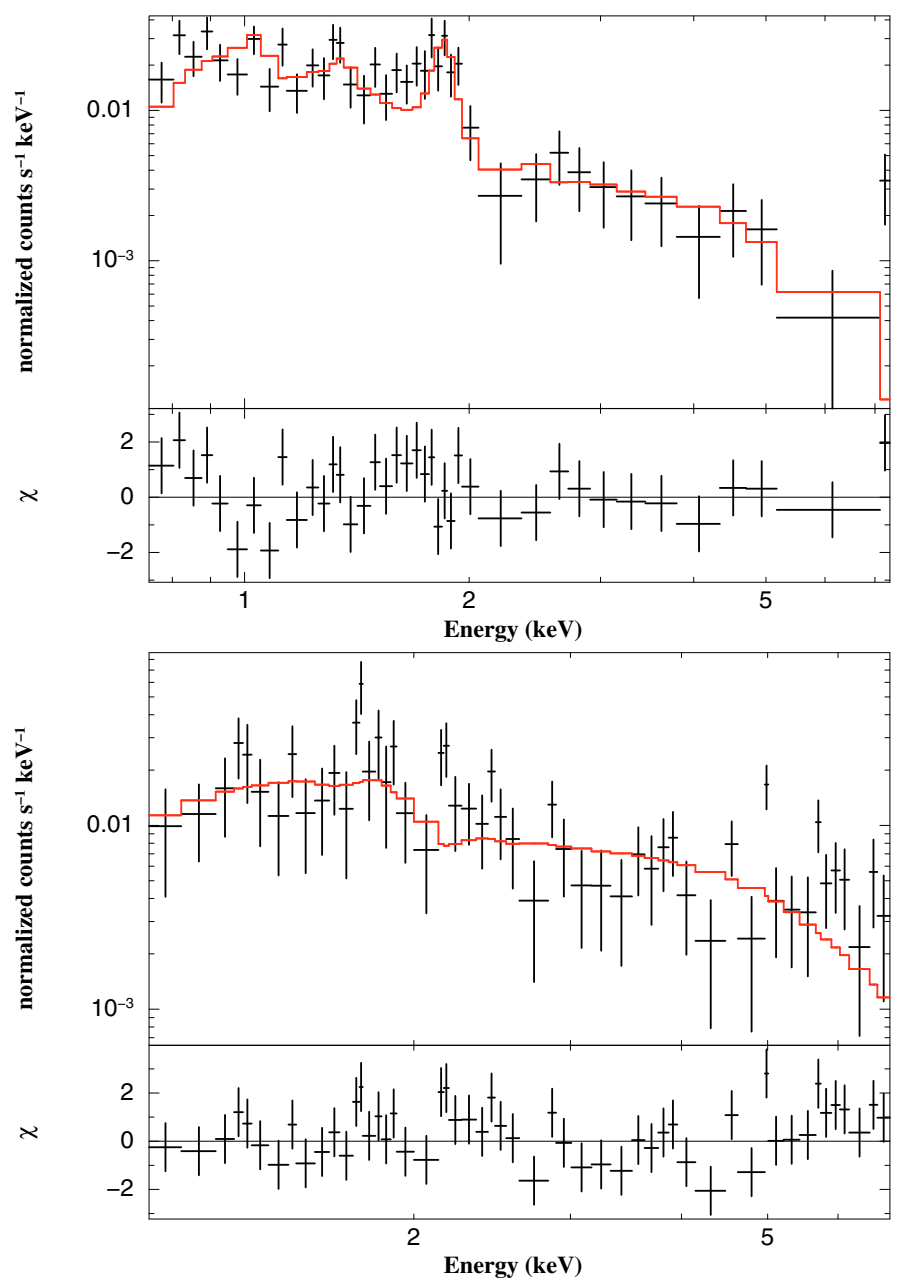

Fig. 3. Top: Chandra spectrum from the outer annulus (175-246") with a 2-temperature non-equilibrium ionization model fit (stepped red line). All parameters are fixed to the values from Ebisawa et al. (2005) except for the surface brightnesses of the two components. Bottom: Chandra spectrum from the inner annulus (55-175") with the spectrum from the outer annulus subtracted as background. The fit is an absorbed powerlaw model (red stepped line). The parameters for both fits are given in Table 1.

of $0.7-10 \mathrm{keV}$ in this specific case. The best-fit values are given in Table 1 (Outer region). The total intrinsic surface flux of the two components is a factor of three lower than the value for the Galactic region observed by Ebisawa et al. (2005). This relation is in good agreement with the ratio between the column densities for both regions, which is $\sim 4$ (Dickey \& Lockman 1990). Assuming that the Galactic column density seen from a certain direction is directly related to the expected flux from a diffuse Galactic component, we conclude that at least $\sim 3 / 4$ of the total excess above particle background observed from the outer region comes from Galactic diffuse emission.

\subsection{Diffuse excess emission connected to Terzan 5}

In this section we focus on the diffuse emission observed from the inner five rings $\left(55-175^{\prime \prime}\right)$. In addition to the radial dependence of the diffuse excess emission, Fig. 2 shows the infrared surface brightness profile (Trager et al. 1995) and the X-ray point-source distribution (King-profile from Heinke et al. 2006). Both profiles are scaled to match the first diffuse X-ray data point, using an exponential fit in the case of the infrared data.
To investigate the nature of the diffuse excess emission observed from the inner region in more detail and to improve the statistical quality, we extracted the combined spectrum from the inner five rings $\left(55-175^{\prime \prime}\right)$. As a first step we fitted the same 2-T NEI model to the NXB subtracted inner spectrum, binned to a minimum of 20 excess counts per bin, as was done for the outer region in the previous section. The resulting surface fluxes of the two components are listed in Table 1 (Inner region). Following the same argument as in the previous section, we estimate that in this case only $\sim 1 / 3$ of the total observed emission is of diffuse Galactic origin. Together with the surface brightness showing a clear radial dependence with respect to the core of Terzan 5, we conclude that a significant part of the observed flux is connected to the GC.

As an estimate for the Galactic diffuse background component, we subtracted the outer (175-246", see previous section) from the inner spectrum. Figure 3 (Bottom) shows the resulting excess spectrum from the inner region, binned to a minimum of 20 excess counts per bin, together with an absorbed power-law model fit. The spectral parameters are collected in Table 1 (Inner region). Fitting a thermal plasma (MEKAL, $\chi_{v}^{2}=1.4(50)$ ) or a thermal bremsstrahlung model (BREMSS, $\chi_{v}^{2}=1.4(50)$ ) to the spectrum gave temperatures $k T>17 \mathrm{keV}$ and $k T>25 \mathrm{keV}$, respectively. The most prominent feature of the Galactic thermal emission observed from the outer region is an emission line, centered on $\sim 1.8 \mathrm{keV}$. Introducing a Gaussian line at that energy or an additional thermal component to the excess spectrum from the inner annulus does not significantly improve the fit $\left(\chi_{v}^{2}=1.2\right)$. Therefore, we conclude that the spectrum from the outer region describes the Galactic diffuse background component sufficiently. The total unabsorbed diffuse excess flux in the $1-7 \mathrm{keV}$ band measured from the inner region above the Galactic background is $F_{\mathrm{X}}=(5.5 \pm 0.8) \times 10^{-13} \mathrm{erg} \mathrm{cm}^{-2} \mathrm{~s}^{-1}$. Assuming a distance of $5.5 \mathrm{kpc}$ the intrinsic luminosity is $L_{\mathrm{X}}=$ $(2.0 \pm 0.3) \times 10^{33} \mathrm{erg} \mathrm{s}^{-1}$.

We found no indication of a variation in the spectral index with increasing radius, when subdividing the inner region into two or more sub-regions. Furthermore, there is no evidence of a directional variation in the index and flux. Table 1 (north, east, south, and west regions) illustrates the result for directional dependence of spectra extracted from pie-shaped regions towards the north, east, south and west with respect to the cluster center (Fig. 1). Their inner and outer radii are 60" and 120", respectively. The latter value was chosen such that the southern region is not truncated by the FoV. As background we again used the spectrum from the outer annulus. A similar result was achieved when the regions were rotated by 45 degrees.

\section{Origin of the diffuse emission}

The present results indicate GC-centered diffuse hard X-ray excess emission above Galactic background, which extends significantly beyond $r_{\mathrm{h}}$. In this section we briefly discuss standard thermal and non-thermal emission scenarios, leaving out more exotic possibilities, as described by, e.g., Domainko \& Ruffert (2005). Throughout this section we use a distance to Terzan 5 of $5.5 \mathrm{kpc}$. The larger distance estimate $(8.7 \mathrm{kpc})$ would increase the energy requirements for the models by a factor of 2.5 .

\subsection{Contribution from unresolved point sources}

The luminosity of unresolved point sources inside $r_{\mathrm{h}}$ has been estimated by Heinke et al. (2006) to $8 \times 10^{32} \mathrm{erg} \mathrm{s}^{-1}$. They 
furthermore determined the spatial surface distribution of X-ray sources in Terzan 5 to $S(r) \propto\left(1+\left(r / r_{\mathrm{c}}\right)^{2}\right)^{(1-3 q) / 2}$ with $q=1.43$. From this distribution we expect to find in the $1-3$ arcmin annulus only $9 \%$ of the luminosity of unresolved point sources within $r_{\mathrm{h}}$. The expected $7 \times 10^{31} \mathrm{erg} \mathrm{s}^{-1}$ is much lower than the measured emission $(2.0 \pm 0.3) \times 10^{33} \mathrm{erg} \mathrm{s}^{-1}$, so we conclude that the contribution from unresolved point sources is negligible.

\subsection{Synchrotron radiation}

One possibility for producing non-thermal emission by relativistic electrons is synchrotron radiation emission (SR), which would radiate at a frequency $v_{\text {syn }}=120\left(\gamma / 10^{4}\right)^{2}(B / 1 \mu \mathrm{G})$ $\sin (\phi) \mathrm{MHz}$, where $B$ is the strength of the magnetic field, $\gamma$ the Lorentz factor of the electrons, and $\phi$ the pitch angle between the magnetic field and the electron velocity (Ok07). Following Ok07, electrons with an energy of $\sim 10^{14} \mathrm{eV}$ would be needed to produce SR emission in typical Galactic magnetic fields of a few $\mu \mathrm{G}$ in the keV regime. The population of MSPs in the center of Terzan 5 was suggested as a continuous source of such highly-energetic electrons (Bednarek \& Sitarek 2007; Venter et al. 2009). These particles propagate to the observed extension of the diffuse emission of $3^{\prime}(4.8 \mathrm{pc})$ on a timescale of $t_{\text {diff }}=3 \times 10^{3} B_{1 \mu \mathrm{G}}$ years, assuming Bohm diffusion (VJ08). The cooling of electrons with energies of $\sim 10^{14} \mathrm{eV}$ in GCs is dominated by SR emission with typical cooling times of $t_{\text {cool }} \approx 3 \times 10^{4} B_{1 \mu \mathrm{G}}^{-2}$ years (VJ08). Assuming an injection spectrum with index -1 , SR cooling, which depends linearly on the energy of the electrons, should change the index to -2 . Since no such steepening of the spectrum is observed at the $2 \sigma$ level, $t_{\text {diff }} \lessgtr t_{\text {cool }}$ is required, which would limit the magnetic field to $\sim 1 \mu \mathrm{G}$ or it would indicate a faster diffusion of electrons. In this scenario, the population of highly energetic electrons has to radiate the observed X-ray luminosity $\left(2 \times 10^{33} \mathrm{erg} \mathrm{s}^{-1}\right)$ on a timescale of $t_{\text {cool }}$, so would require a total energy in these electrons of $1.8 \times 10^{45} B_{1 \mu \mathrm{G}}^{-2}$ erg. Associated IC radiation in the TeV energy range should be detectable in spatial coincidence in the case of low magnetic fields $B \lesssim$ few $\mu \mathrm{G}$, providing a test for this scenario (VJ08).

\subsection{Inverse Compton emission}

Non-thermal X-ray emission in GCs can also be produced by IC up-scattering of star-light photons by mildly relativistic electrons (Krockenberger \& Grindlay 1995). The bow shock of the GC could provide these electrons (Ok07). The power of IC radiation $P_{\mathrm{IC}}$ emitted by a single electron is given by $P_{\mathrm{IC}}=$ $4 / 3 \sigma_{\mathrm{T}} c \gamma^{2} u_{\mathrm{rad}}$, where $\sigma_{\mathrm{T}}$ is the Thomson cross section, $c$ is the speed of light, $\gamma$ the energy of the electrons, and $u_{\mathrm{ph}}$ the density of the target photon field (Krockenberger \& Grindlay 1995). Therefore the intensity of IC emission is directly related to the energy density of the target photon field. GCs exhibit a very high stellar density in their core region, which decreases rapidly in their outskirts, resulting in a centrally peaked photon field as indicated with the distribution of the infrared surface brightness in Fig. 2. The diffuse X-ray emission presented in this paper exhibits a surface brightness profile that is roughly similar to this proxy of the density of the photon field, consistent with IC emission. The energy density $u_{\text {rad }}$ of the stellar photon field scaling with $u_{\mathrm{rad}} \approx L_{\mathrm{star}} /\left(4 \pi \mathrm{r}^{2} \mathrm{c}\right)$ is about $40 \mathrm{eV} / \mathrm{cm}^{3}$ and $5 \mathrm{eV} / \mathrm{cm}^{3}$, at distances of $1^{\prime}$ and $3^{\prime}$, respectively, from the center of the GC. For the quantitative estimate of the total energy in electrons we adopt the model of Krockenberger \& Grindlay (1995) giving $5 \times 10^{49}\left(u_{\mathrm{rad}} / 40 \mathrm{eV} \mathrm{cm}^{-3}\right) \mathrm{erg}$. In this scenario the X-ray emission should be accompanied by potentially detectable SR emission in the radio band.

\subsection{Non-thermal bremsstrahlung}

One additional emission process of non-thermal X-rays is nonthermal bremsstrahlung, which is produced when energetic electrons are deflected by protons and nuclei. In this scenario the flux of the emission should follow the distribution of target material. The Galactic density profile of ISM perpendicular to the Galactic plane at the relevant galactocentric distances of $1-3 \mathrm{kpc}$ was constrained as a single Gaussian with a full width at half maximum of less than $200 \mathrm{pc}$ and virtually no gas above $400 \mathrm{pc}$ (Lockman 1984). At a distance of $5.5 \mathrm{kpc}$, Terzan 5 would be at an offset above the disk of $160 \mathrm{pc}$ and thus in an ambient gas density of a few times $0.1 \mathrm{~cm}^{-3}$. The total energy in nonthermal electrons required for the emission of $2 \times 10^{33} \mathrm{erg} \mathrm{s}^{-1}$ of diffuse X-ray emission would be about $9 \times 10^{49}\left(n_{\mathrm{H}} / 0.1 \mathrm{~cm}^{-3}\right) \mathrm{erg}$ if an electron energy of $20 \mathrm{keV}$ is assumed (Ok07). In contrast to the asymmetric morphology detected by Ok07 for GCs in a bow-shock scenario, we did not find evidence of a non-uniform shape of the excess emission from Terzan 5. This scenario could be tested by the presence of target material in the environment of the GC. Target material in the form of molecular clouds could be probed by carefully examining molecular emission lines that are shifted by the relative Galactic rotation velocity at the physical location of Terzan 5 .

\subsection{Thermal contribution}

The very high fitted temperature $(>15 \mathrm{keV})$ of the diffuse X-ray emission would suggest a non-thermal origin. However, at least a thermal contribution to the total excess cannot be excluded at this point. If thermal bremsstrahlung is presumed as the emission mechanism, the temperature of the plasma can be estimated from the X-ray luminosity, the volume of the emission region, and the density of the plasma (Krockenberger \& Grindlay 1995). With the radius of the emission region set to $5 \mathrm{pc}$ (which corresponds to $188^{\prime \prime}$ at a distance of $5.5 \mathrm{kpc}$ ), this leads to

$\left(\frac{T}{10^{7} \mathrm{~K}}\right) \approx\left(\frac{L_{\mathrm{X}}^{\mathrm{th}}}{1.3 \times 10^{33}\left(n / 0.1 \mathrm{~cm}^{-3}\right)^{2}}\right)^{2}$.

Assuming that the observed emission is entirely thermal, i.e., $L_{\mathrm{X}}^{\text {th }}=L_{\mathrm{X}}$, provides an upper bound for the temperature of the plasma. It appears that this upper bound strongly depends on the plasma density. For a typical density at the GC position of $0.1 \mathrm{~cm}^{-3}$ (see Sect. 3.4), the upper bound on the temperature is about $10^{7} \mathrm{~K}(\approx 1 \mathrm{keV})$. Only for densities lower than $0.05 \mathrm{~cm}^{-3}$ can the temperature exceed $15 \mathrm{keV}$.

To heat plasma to such high temperatures, strong shocks would be indispensable. The remnants of catastrophic events may release such strong shocks (see, e.g., Acero et al. 2007, for a remnant of a supernova Ia and Domainko \& Ruffert 2005, for remnants of compact binary mergers). It was proposed that Terzan 5 may host the required mergers; e.g., Shara \& Hurley (2002) for white dwarf mergers and Grindlay et al. (2006) for neutron star - neutron star mergers. However, even supernova remnants may have difficulty producing such high temperatures, because even the hot, thermal plasma in the young remnant of the type Ia supernova remnant SN 1006 reaches a temperature 
of about $2 \mathrm{keV}$ (e.g. Acero et al. 2007), significantly cooler than the temperature found for the thermal fit to the diffuse emission in Terzan 5.

If the diffuse X-ray emission is indeed thermal, it could also originate in principle from a background galaxy cluster that by chance coincides with the core of Terzan 5. Since galaxy clusters with temperatures $>10 \mathrm{keV}$ are very rare (e.g. Reiprich \& Böhringer 2002), such a correlation appears rather unlikely.

From the available data, a contribution from thermal emission processes to the measured flux cannot be ruled out, but it is not likely to represent the dominant fraction.

\section{Conclusions}

We discovered diffuse hard X-ray emission from the GC Terzan 5 with a photon index of about 1 and a peak flux density profile centered on the cluster core. The hard photon index makes a purely thermal emission scenario unlikely. Energetics would favor an SR scenario as the origin of the emission and would challenge simple IC and non-thermal Bremsstrahlung models generated by electrons accelerated by the bow shock of the GC. However, no simple model is clearly preferred to explain the observed emission, as expected from the limited statistics provided by the available X-ray dataset. Additional X-ray observations, detailed multi-wavelength informations, as well as refined modeling are needed to accurately interpret the unique properties of the diffuse X-ray radiation in Terzan 5.

Acknowledgements. This research has made use of data obtained from the Chandra Data Archive and software provided by the Chandra X-ray Center (CXC) in the application packages CIAO and ChIPS. We thank the referee for the very constructive feedback.

\section{References}

Acero, F., Ballet, J., \& Decourchelle, A. 2007, A\&A, 475, 883

Arnaud, K. A. 1996, in Astronomical Data Analysis Software and Systems V, ASP Conf. Ser., 101, 17

Bednarek, W., \& Sitarek, J. 2007, MNRAS, 377, 920

Cohn, H. N., Lugger, P. M., Grindlay, J. E., \& Edmonds, P. D. 2002, ApJ, 571, 818

Dickey, J. M., \& Lockman, F. J. 1990, ARA\&A, 28, 215

Domainko, W., \& Ruffert, M. 2005, A\&A, 444, L33

Ebisawa, K., Tsujimoto, M., Paizis, A., et al. 2005, ApJ, 635, 214

Garmire, G. P., Bautz, M. W., Ford, P. G., Nousek, J. A., \& Ricker, Jr., G. R.

2003, in Society of Photo-Optical Instrumentation Engineers (SPIE) Conf. Ser., ed. J. E. Truemper \& H. D. Tananbaum, 4851, 28

Grindlay, J., Portegies Zwart, S., \& McMillan, S. 2006, Nature Physics, 2, 116

Harris, W. E. 1996, AJ, 112, 1487

Hartwick, F. D. A., Grindlay, J. E., \& Cowley, A. P. 1982, ApJ, 254, L11

Heinke, C. O., Wijnands, R., Cohn, H. N., et al. 2006, ApJ, 651, 1098

Ivanova, N., Fregeau, J. M., \& Rasio, F. A. 2005, in Binary Radio Pulsars, ed. F. A. Rasio \& I. H. Stairs, Astronomical Society of the Pacific Conf. Ser., 328, 231

Kaneda, H., Makishima, K., Yamauchi, S., et al. 1997, ApJ, 491, 638

Krockenberger, M., \& Grindlay, J. E. 1995, ApJ, 451, 200

Lockman, F. J. 1984, ApJ, 283, 90

Markevitch, M., Bautz, M. W., Biller, B., et al. 2003, ApJ, 583, 70

Masai, K. 1984, Ap\&SS, 98, 367

Okada, Y., Kokubun, M., Yuasa, T., \& Makishima, K. 2007, PASJ, 59, 727

Ortolani, S., Barbuy, B., Bica, E., Zoccali, M., \& Renzini, A. 2007, A\&A, 470, 1043

Ransom, S. M. 2008, in IAU Symposium, ed. E. Vesperini, M. Giersz, \& A. Sills, 246, 291

Reiprich, T. H., \& Böhringer, H. 2002, ApJ, 567, 716

Shara, M. M., \& Hurley, J. R. 2002, ApJ, 571, 830

Trager, S. C., King, I. R., \& Djorgovski, S. 1995, AJ, 109, 218

Venter, C., \& de Jager, O. C. 2008, in AIP Conf. Ser., ed. F. A. Aharonian, W. Hofmann, \& F. Rieger, 1085, 277

Venter, C., De Jager, O. C., \& Clapson, A. 2009, ApJ, 696, L52

Weisskopf, M. C., Brinkman, B., Canizares, C., et al. 2002, PASP, 114, 1

Wijnands, R., Heinke, C. O., Pooley, D., et al. 2005, ApJ, 618, 883

Yuasa, T., Nakazawa, K., \& Makishima, K. 2009, PASJ, 61, 1107 